\title{
Distal femoral bone mineral density decreases following patellofemoral arthroplasty: 1-year follow-up study of 14 patients
}

\author{
Hans-Peter W van Jonbergen*1, Kenneth Koster², Luc Labey³, Bernardo Innocenti ${ }^{3}$ and Albert van Kampen ${ }^{4}$
}

\begin{abstract}
Background: The bone mineral density (BMD) of the distal femur decreases by $16-36 \%$ within one year after total knee arthroplasty (TKA) because of the femoral component's stress-shielding effect. The aim of this prospective study was to determine the quantitative change from the baseline BMD in the distal femur 1 year after patellofemoral arthroplasty using dual-energy X-ray absorptiometry (DXA).

Methods: Between December 2007 and December 2008, 14 patients had patellofemoral arthroplasty for isolated patellofemoral osteoarthritis. Distal femoral BMD was assessed using DXA in 2 regions of interest (ROI) on the lateral view 2 weeks before and 12 months after patellofemoral arthroplasty. The contra-lateral knee was used as a control, with BMD measurements performed in identical ROls.

Results: The mean change from baseline BMD in the operated knees after 1 year was $-0.169 \mathrm{~g} / \mathrm{cm}^{2}(95 \% \mathrm{Cl}:-0.293$ to $\left.0.046 \mathrm{~g} / \mathrm{cm}^{2}\right)$ behind the anterior flange $(-15 \%)$, and $-0.076 \mathrm{~g} / \mathrm{cm}^{2}\left(95 \% \mathrm{Cl}:-0.177\right.$ to $\left.0.024 \mathrm{~g} / \mathrm{cm}^{2}\right)$ in the supracondylar area $1 \mathrm{~cm}$ above the prosthesis $(-8 \%)(p=0.01$ and $p=0.13$, respectively). The mean change from baseline BMD in the non-operated knees after 1 year was $0.016 \mathrm{~g} / \mathrm{cm}^{2}\left(95 \% \mathrm{Cl}:-0.152\right.$ to $\left.0.185 \mathrm{~g} / \mathrm{cm}^{2}\right)$ behind the anterior flange (2\%), and $0.023 \mathrm{~g} / \mathrm{cm}^{2}\left(95 \% \mathrm{Cl}:-0.135\right.$ to $\left.0.180 \mathrm{~g} / \mathrm{cm}^{2}\right)$ in the supracondylar area $1 \mathrm{~cm}$ above the prosthesis $(2 \%)(p=0.83$, and $p=$ 0.76 , respectively).
\end{abstract}

Conclusions: Our findings suggest that patellofemoral arthroplasty results in a statistically significant decrease in BMD behind the anterior flange.

\section{Background}

After total knee arthroplasty (TKA), the bone mineral density (BMD) of the distal femur decreases by $16-36 \%$ within one year because of the femoral component's stress-shielding effect [1-7]. Although the femoral component in patellofemoral arthroplasty is smaller than in TKA, the mechanical loading, and consequently the stress distribution of the distal femoral bone, is altered compared with the physiological situation. This can lead to bone remodeling, resulting in decreased BMD behind the anterior flange of the femoral component. In TKA, bone loss in the distal anterior femur can lead to supracondylar fractures or loosening of the implant, and may induce difficulties during revision arthroplasty [8,9].

\footnotetext{
* Correspondence: vanjonbergen@dz.nl

${ }^{1}$ Department of Orthopedic Surgery, Deventer Hospital, Nico Bolkesteinlaan 75, 7416 SE Deventer, The Netherlands

Full list of author information is available at the end of the article
}

Since patellofemoral arthroplasty is typically used in younger patients, conversion to TKA after painful femorotibial osteoarthritis develops will eventually be performed in a relatively large proportion of patients [10]. Although the clinical outcome of TKA done later does not appear to be influenced by prior patellofemoral arthroplasty [11], the results of such a revision may, however, be compromised by loss of bone stock.

To date, no clinical studies have addressed the possible decrease in distal femoral BMD as a parameter of bone remodeling following patellofemoral arthroplasty. We hypothesized that because of the relative small size there is no significant stress-shielding effect behind the femoral component of a patellofemoral prosthesis resulting in a decrease in BMD in the distal femur. The primary objective was, therefore, to determine the change from baseline in the BMD behind the anterior flange 1 year after 
patellofemoral arthroplasty using dual-energy X-ray absorptiometry (DXA).

\section{Methods}

In 2007, we initiated a prospective study to investigate the distal femoral BMD using DXA in patients undergoing patellofemoral arthroplasty. All patients who were planned for patellofemoral arthroplasty for isolated patellofemoral osteoarthritis at Deventer Hospital, Deventer, The Netherlands, were evaluated for inclusion in the study. Patients with known rheumatic, renal, hepatic, or gastrointestinal disease, and patients using medication that interferes with mineral metabolism (i.e. treatment for osteoporosis or long-term steroid therapy) were excluded from the study. Additionally, patients with a previous TKA or patellofemoral arthroplasty of the contra-lateral knee were excluded. The study was approved by the Regional Ethics Committee (NL16145.075.07, December 2007) and Institutional Review Board.

Sample size was calculated using estimates of mean femoral BMD and standard deviation (SD) behind the anterior flange after TKA [1]. The reported mean BMD behind the anterior flange of a total knee prosthesis in the replaced knee was $0.94 \mathrm{~g} / \mathrm{cm}^{2}(0.31)$, and $1.25 \mathrm{~g} / \mathrm{cm}^{2}$ (0.30) in the contra-lateral, non-replaced knee [1]. A group sample size of 13 patients achieves $95 \%$ power to detect a difference of $0.31 \mathrm{~g} / \mathrm{cm}^{2}$ between the null hypothesis that both group means are $1.25 \mathrm{~g} / \mathrm{cm}^{2}$, and the alternative hypothesis that the mean of group 2 (replaced knee) is $0.94 \mathrm{~g} / \mathrm{cm}^{2}$ with known group SDs of $0.31 \mathrm{~g} / \mathrm{cm}^{2}$ and $0.30 \mathrm{~g} / \mathrm{cm}^{2}$ and with a significance level (alpha) of 0.05 using a two-tailed paired t-test (PASS 2008, NCSS software, Kaysville, Utah).

Between December 2007 and December 2008, 2 orthopedic surgeons who performed patellofemoral arthroplasty at Deventer Hospital recruited 14 patients. All patients provided written informed consent. All eligible patients were preoperatively assessed by 1 of the 2 participating orthopedic surgeons, who completed the Knee Society Knee Score (KSKS) and the Knee Society Functional Score (KSFS). The Dutch version of the Western Ontario and McMaster Universities Osteoarthritis Index 3.1 (WOMAC) was completed by all patients. Measurement of the BMD in the distal femur was performed using DXA in the lateral view (GE Lunar Prodigy system, General Electrics, Oldelft Benelux B.V., Delft/ Veenendaal, The Netherlands) 2 weeks before patellofemoral arthroplasty and 12 months after arthroplasty. Measurements of a calibration phantom were performed each day before scanning the patients. All measurements were made by an independent radiographic technician. Both the scanning procedure and positioning of the patients and knees were standardized, with the patient in the lateral decubitus position and the knee flexed 15-30 degrees to obtain a true lateral scan. Two regions of interest (ROI) were selected; one in the distal anterior area just behind the anterior flange of the prosthesis (centered between the tip of the fixation peg and the proximal end of the prosthesis) (ROI 1), and the other more proximally, in the supracondylar area $1 \mathrm{~cm}$ superior to the anterior flange of the femoral component (ROI 2) (Figure 1). ROI 2 was selected as a reference ROI above the prosthesis, where stress-shielding was assumed to be negligible. The measured area of each ROI was $1 \times 1 \mathrm{~cm}$. The contra-lateral, non-operated knee was used as a control, with BMD measurements in identical ROIs. We employed knee-specific software in all cases.

Two similarly experienced surgeons at our institution performed patellofemoral arthroplasty with the currently commercially available Richards type II prosthesis (Smith \& Nephew Inc., Memphis, Tennessee). Surgery was performed under pneumatic tourniquet control and antibi-

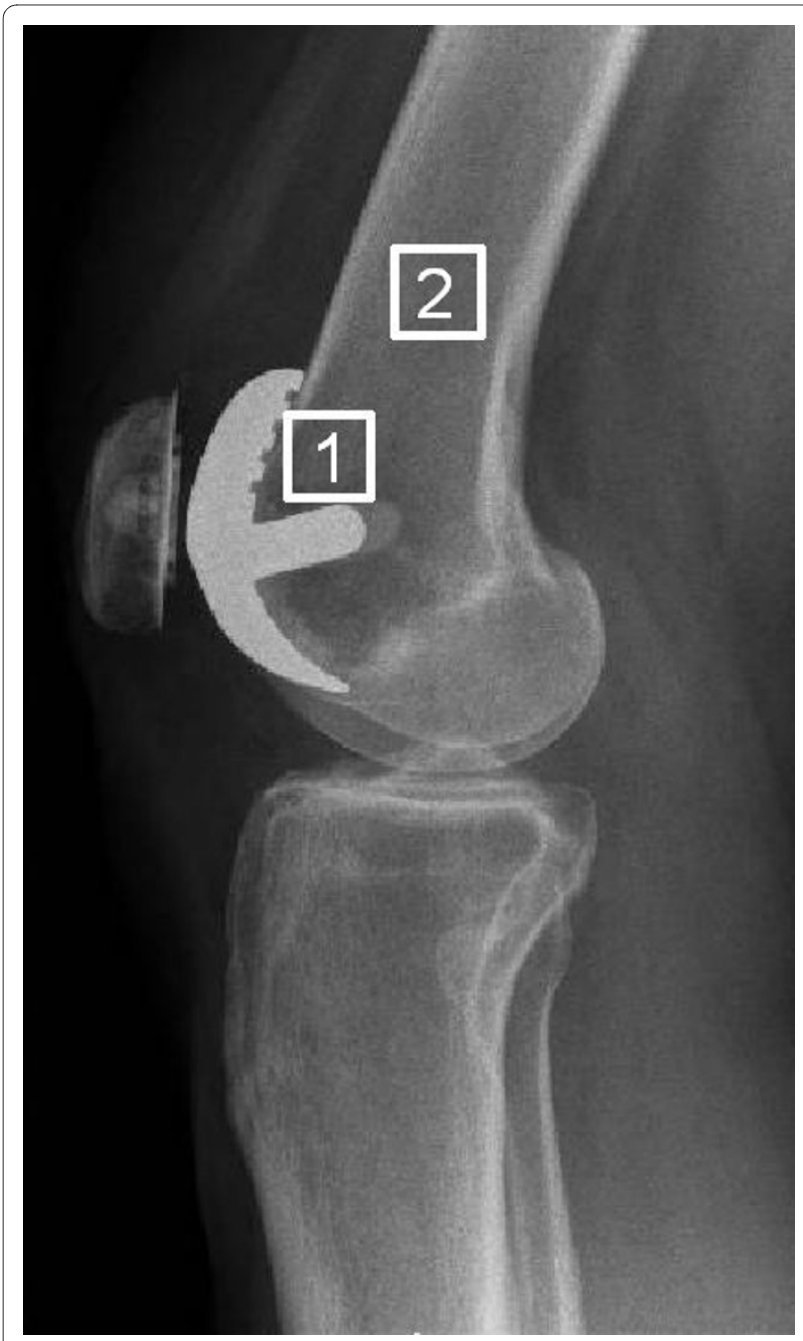

Figure 1 Location of regions of interest (ROI) on a lateral radiograph of a right knee. 
otic prophylaxis using intravenous Cefazoline $1 \mathrm{~g}, 3$ times daily, for the first 24 hours with the first dose administered 30 minutes before application of the tourniquet. All operations were performed in an identical manner according to the manufacturers' instruction, as described elsewhere [10]. No intramedullary guiding rod was used during surgery. All 14 patients received the same postoperative treatment. We allowed patients protected weight bearing with crutches immediate after surgery, and full unrestricted weight bearing was allowed 6 weeks after surgery. All patients routinely received antithrombotic prophylaxis with a low-molecular-weight heparin (Fragmin) for 6 weeks.

All patients had regular clinical follow-ups at 2 and 8 weeks to evaluate wound healing and rehabilitation, DXA was not performed at these follow-up visits. At the 1-year follow-up, patients were clinically assessed using the KSKS and KSFS, and were asked to complete the WOMAC questionnaire. During follow-up, the radiological examinations consisted of 2 radiographs (anteroposterior standing and lateral non-weight bearing) performed 6 weeks and one year post surgery (Figure 2). Radiological findings were reported using the Knee Society total knee arthroplasty roentgenographic evaluation and scoring system [12].

All pertinent data were entered in a spreadsheet program and analyzed using PASW Statistics 18 software (SPSS Inc, Chicago, Illinois). We performed descriptive analysis using the mean and standard deviation for continuous variables, and frequencies for categorical variables. The $95 \%$ confidence intervals (CI) were calculated for the absolute changes in BMD from baseline. The twotailed paired $t$-test was used to analyze for differences in preoperative and postoperative BMD. A linear regression model was used to evaluate for influence of BMI, age, and

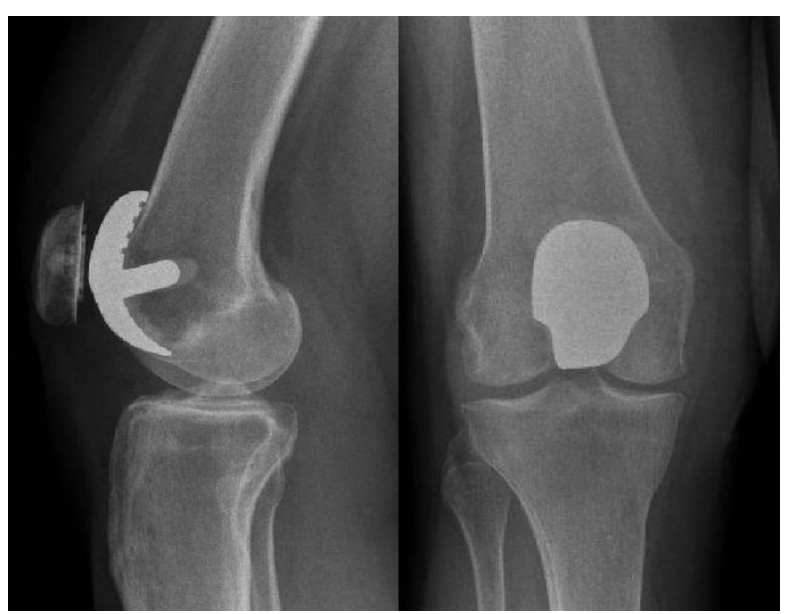

Figure 2 Lateral (left) and anteroposterior (right) radiographs of a right knee one year after patellofemoral arthroplasty. sex on change in BMD from baseline. A p-value of less than 0.05 was considered significant in all the tests.

\section{Results}

Between December 2007 and December 2008, 14 patients had unilateral patellofemoral arthroplasty, receiving the Richards type II patellofemoral prosthesis. All 14 patients were available for the one year follow-up. The patient's demographic data are presented in Table 1.

Mean KSKS improved from 61 (range, 50 to 78) preoperatively to 88 (range, 60 to 100) one year after surgery (p $<0.001$ ). Mean KSFS improved from 65 (range, 50 to 80 ) preoperatively to 86 (range, 50 to 100 ) one year after surgery $(\mathrm{p}=0.004)$. The mean preoperative WOMAC scores improved from 50 (range, 22 to 69 ) to 23 (range, 4 to 39) one year after surgery $(\mathrm{p}<0.001)$. No complications were noted from the surgical procedure within the study period. The 1-year radiographic follow-up showed that all prostheses were in good alignment without radiolucent lines or osteolysis.

Results of the BMD measurements are summarized in Table 2. In the operated knees, there was a 15\% decrease in mean BMD at 12 months in ROI $1(\mathrm{p}=0.01)$, and an $8 \%$ decrease in ROI 2 ( $\mathrm{p}=0.13)$. In the non-operated knees, there was a $2 \%$ increase in the mean BMD at 12 months in both ROI 1 and ROI 2 ( $\mathrm{p}=0.83$ and $\mathrm{p}=0.76$, respectively).

Regression analysis of the change from baseline BMD for both regions of interest against BMI (regression coefficient $=0.017, \mathrm{p}=0.3$ ), age (regression coefficient $=$ $0.002, \mathrm{p}=0.8$ ), and sex (regression coefficient $=-0.063, \mathrm{p}$ $=0.6$ ) demonstrated no significant relationships.

\section{Discussion}

The results of our prospective, 1-year DXA study demonstrate a statistically significant $15 \%$ decrease in BMD behind the anterior flange of the femoral component during the first year after patellofemoral arthroplasty. To our knowledge, there has been no previous study that

Table 1: Patient characteristics.

\section{Characteristic}

\begin{tabular}{ll}
\hline Number of knees & 14 \\
Side (right: left) & $7: 7$ \\
Mean (SD) age at surgery & $53(10)$ years \\
Sex (female: male) & $9: 5$ \\
Mean (SD) Height & $175(5) \mathrm{cm}$ \\
Mean (SD) Weight & $87(13) \mathrm{kg}$ \\
Mean (SD) body mass index & $28(4) \mathrm{kg} / \mathrm{m}^{2}$
\end{tabular}

Continuous values are given as the mean with standard deviation in parentheses. 
Table 2: Bone mineral density $\left(B M D, g / \mathrm{cm}^{2}\right)$ measured in the distal femur before $(t=0)$ and 12 months after $(t=12)$ patellofemoral arthroplasty.

\begin{tabular}{|c|c|c|c|c|c|}
\hline & BMD $(t=0)$ & $B M D(t=12)$ & $\begin{array}{l}\text { Change from } \\
\text { baseline }\end{array}$ & $\begin{array}{l}\text { 95\% } \mathrm{Cl} \text { for the } \\
\text { difference }\end{array}$ & p-value \\
\hline \multicolumn{6}{|c|}{ Operated knee } \\
\hline ROI 1 & $1.106(0.229)$ & $0.937(0.362)$ & $-0.169(0.204)$ & -0.293 to -0.046 & 0.01 \\
\hline ROI 2 & $0.981(0.272)$ & $0.905(0.261)$ & $-0.076(0.167)$ & -0.177 to 0.024 & 0.13 \\
\hline \multicolumn{6}{|c|}{$\begin{array}{l}\text { Non-operated } \\
\text { knee }\end{array}$} \\
\hline ROI 1 & $1.099(0.266)$ & $1.115(0.315)$ & $0.016(0.251)$ & -0.152 to 0.185 & 0.83 \\
\hline ROI 2 & $0.930(0.175)$ & $0.952(0.266)$ & $0.023(0.234)$ & -0.135 to 0.180 & 0.76 \\
\hline
\end{tabular}

Continuous values are given as the mean with standard deviation in parentheses.

$\mathrm{Cl}$, confidence interval; $\mathrm{ROI}$, region of interest.

attempted to use DXA measurements to evaluate changes in the distal femoral BMD after patellofemoral arthroplasty. Several investigators reported the results of BMD measurements after TKA and demonstrated periprosthetic bone loss of up to $36 \%$ adjacent to the implants [17]. The general BMD as measured in hip and spine, or the $\mathrm{BMD}$ in the contra-lateral knee, did not change after arthroplasty [1-3,7].

Finite-element models were used to determine whether patterns of bone resorption behind the femoral component in TKA could be explained by strain-adaptive bone remodeling $[13,14]$. With a bonded femoral component, the predicted long-term bone loss would occur at the most distal part of the femur and behind the anterior part of the prosthesis [13]. These findings are in agreement with the results of clinical DXA studies, which observed loss of BMD behind the anterior flange of the femoral component in TKA [1-7]. Our results show that comparable distal femoral bone loss occurs following patellofemoral arthroplasty. This suggests that the stressshielding effect is similar to that which occurs after TKA, even with the smaller femoral component of the patellofemoral prosthesis (Figure 2). However, the clinical results do not seem to be compromised by the observed loss of bone. In a recent study, the long-term outcomes of the Richards type II patellofemoral arthroplasty were reported [10]. The most common reasons for conversion in this series were progression of femorotibial osteoarthritis and revision for malpositioning that resulted in catching and instability. Loosening of the femoral component was not observed, which is in accordance with the literature. Furthermore, the clinical outcome of later TKA does not appear to be influenced by prior patellofemoral arthroplasty $[11,15]$. No technical difficulties were experienced during conversion, and the condylar support in each knee was uncompromised.
The observed decrease in the BMD in the supracondylar reference ROI 2 was not statistically significant. Other investigators demonstrated an 8\% decrease in the BMD in this diaphyseal ROI at 1 year after total knee arthroplasty $[5,7]$. Soininvaara et al. suggested that this less pronounced bone loss represented both operation-related and postoperative immobilization-induced bone loss, because age-related bone loss is minor [5]. However, physical activity had improved in our patients, as demonstrated by the improvement in KSFS and WOMAC scores.

The current study has some limitations that should be noted. Follow-up examinations were performed at 1 year after surgery. With TKA, several investigators report that no additional remodeling occurs after 6-12 months [2,57], while others stated that loss of BMD stabilizes within 2 years $[1,16]$. Finite element models predict that bone resorption may continue after 2 years [13]. Thus, it is possible that a further decrease in BMD occurs in our patients. Another limitation is that we performed no precision measurements in our relatively small series. Therefore, we assumed a precision similar to those reported by others using the same Lunar Prodigy system, albeit with possible software differences. The reported precision for BMD measurements on the lateral view of the anterior femur ranges from $1.3 \%$ to $3.6 \%[3,4,7,17,18]$. Because of the relatively small size of the patellofemoral prosthesis, the ROI behind the anterior flange was also necessarily small, which may have resulted in weakened precision [17].

Although our study protocol excluded medical conditions that could have affected the BMD, it is possible that other conditions may have existed that influenced the results. None of the patients had a severe medical disability that limited the ability to walk, or had a disabling disease involving other joints of the lower extremities. We did not assess the amount of physical activity at one-year 
follow-up and were therefore not able to evaluate for a relationship with change from baseline BMD.

\section{Conclusions}

Our findings suggest that Richards type II patellofemoral arthroplasty results in a statistically significant decrease in BMD behind the anterior flange. Newer designs, such as the Journey patellofemoral prosthesis (Smith \& Nephew) and the Zimmer Gender Solutions PFJ system, employ a broader trochlear component. In theory, the geometry, size, and material properties may result in different patterns of stress shielding. Future studies should be aimed at evaluating BMD changes in these newer patellofemoral prostheses.

\section{Competing interests}

Both $L L$ and $B I$ wish to note that they are employees of the European Centre for Knee Research, Smith \& Nephew, Belgium.

\section{Authors' contributions}

HPWVJ initiated the study. HPWVJ and KK collected and analyzed the data. HPWVJ wrote the manuscript under the supervision of $L L, B I$, and AvK. All authors read and approved the final manuscript.

\section{Acknowledgements}

The authors are grateful to Ms. Kay Galbraith, radiographic technician, for her invaluable assistance in performing the DXA measurements.

\section{Author Details}

'Department of Orthopedic Surgery, Deventer Hospital, Nico Bolkesteinlaan 75, 7416 SE Deventer, The Netherlands, ${ }^{2}$ Department of Radiology, Deventer Hospital, Nico Bolkesteinlaan 75, 7416 SE Deventer, The Netherlands, 3European Centre for Knee Research, Smith \& Nephew, Technologielaan 11 Bis, 3001 Leuven, Belgium and ${ }^{4}$ Department of Orthopaedic Surgery and Orthopaedic Research Laboratory, Radboud University Nijmegen Medical Center, PO Box 9101, 6500 HB Nijmegen, The Netherlands

Received: 13 January 2010 Accepted: 20 April 2010 Published: 20 April 2010

\section{References}

1. Abu-Rajab RB, Watson WS, Walker B, Roberts J, Gallacher SJ, Meek RM: Peri-prosthetic bone mineral density after total knee arthroplasty. Cemented versus cementless fixation. J Bone Joint Surg Br 2006, 88:606-613

2. Karbowski A, Schwitalle M, Eckardt A, Heine J: Periprosthetic bone remodelling after total knee arthroplasty: early assessment by dual energy X-ray absorptiometry. Arch Orthop Trauma Surg 1999, 119:324-326.

3. Liu TK, Yang RS, Chieng PU, Shee BW: Periprosthetic bone mineral density of the distal femur after total knee arthroplasty. Int Orthop 1995, 19:346-351.

4. Petersen MM, Olsen C, Lauritzen JB, Lund B: Changes in bone mineral density of the distal femur following uncemented total knee arthroplasty. J Arthroplasty 1995, 10:7-11.

5. Soininvaara TA, Miettinen HJ, Jurvelin JS, Suomalainen OT, Alhava EM, Kroger HP: Periprosthetic femoral bone loss after total knee arthroplasty: 1-year follow-up study of 69 patients. Knee 2004, 11:297-302.

6. Spittlehouse AJ, Getty CJ, Eastell R: Measurement of bone mineral density by dual-energy $X$-ray absorptiometry around an uncemented knee prosthesis. J Arthroplasty 1999, 14:957-963.

7. van Loon CJ, Oyen WJ, de Waal Malefijt MC, Verdonschot N: Distal femoral bone mineral density after total knee arthroplasty: a comparison with general bone mineral density. Arch Orthop Trauma Surg 2001, 121:282-285.
8. van Loon CJ, de Waal Malefijt MC, Buma P, Verdonschot N, Veth RP: Femoral bone loss in total knee arthroplasty. A review. Acta Orthop Belg 1999, 65:154-163

9. Hernigou P, Mathieu G, Filippini P, Demoura A: Facteurs du risque de fracture du fémur distal dans les prothèses totales du genou: Étude de 32 fractures per et postopératoires. Rev Chir Orthop Reparatrice Appar Mot 2006, 92:140-147.

10. van Jonbergen H-PW, Werkman DM, Barnaart LF, van Kampen A: Longterm outcomes of patellofemoral arthroplasty. Journal of Arthroplasty 2010 in press

11. van Jonbergen H-PW, Werkman DM, van Kampen A: Conversion of patellofemoral arthroplasty to total knee arthroplasty: A matched case-control study of 13 patients. Acta Orthop 2009, 80:62-66

12. Ewald FC: The Knee Society total knee arthroplasty roentgenographic evaluation and scoring system. Clin Orthop Relat Res 1989, 248:9-12.

13. Van Lenthe GH, de Waal Malefijt MC, Huiskes R: Stress shielding after total knee replacement may cause bone resorption in the distal femur. J Bone Joint Surg Br 1997, 79:117-122.

14. Tissakht M, Ahmed AM, Chan KC: Calculated stress-shielding in the distal femur after total knee replacement corresponds to the reported location of bone loss. J Orthop Res 1996, 14:778-785.

15. Lonner JH, Jasko JG, Booth RE Jr: Revision of a failed patellofemoral arthroplasty to a total knee arthroplasty. J Bone Joint Surg Am 2006, 88:2337-2342.

16. Cameron HU, Cameron G: Stress-relief osteoporosis of the anterior femoral condyles in total knee replacement. A study of 185 patients. Orthop Rev 1987, 16:449-456

17. Soininvaara T, Kroger $H$, Jurvelin JS, Miettinen $H$, Suomalainen O, Alhava E: Measurement of bone density around total knee arthroplasty using fan-beam dual energy X-ray absorptiometry. Calcif Tissue Int 2000 67:267-272

18. Trevisan C, Bigoni M, Denti M, Marinoni EC, Ortolani S: Bone assessment after total knee arthroplasty by dual-energy X-ray absorptiometry: analysis protocol and reproducibility. Calcif Tissue Int 1998, 62:359-361.

Pre-publication history

The pre-publication history for this paper can be accessed here: http://www.biomedcentral.com/1471-2474/11/74/prepub

doi: 10.1186/1471-2474-11-74

Cite this article as: van Jonbergen et al., Distal femoral bone mineral density decreases following patellofemoral arthroplasty: 1-year follow-up study of 14 patients BMC Musculoskeletal Disorders 2010, 11:74

\section{Submit your next manuscript to BioMed Central} and take full advantage of:

- Convenient online submission

- Thorough peer review

- No space constraints or color figure charges

- Immediate publication on acceptance

- Inclusion in PubMed, CAS, Scopus and Google Scholar

- Research which is freely available for redistribution 